\title{
The use of Galileo signals for time transfer metrology
}

\author{
Pierre Uhrich and Philip Tuckey
}

LNE-SYRTE, Observatoire de Paris, LNE, CNRS, UPMC, 61 avenue de l'Observatoire, 75014 Paris, France

\begin{abstract}
We briefly summarise the planned Galileo navigation system signals and their potential application in time and frequency metrology.
\end{abstract}

Keywords. time

Galileo is the global navigation satellite system (GNSS) currently being developed by the European Union, the European Space Agency and partners. Two experimental satellites are currently in operation, the in orbit validation phase involving 4 new satellites is planned to commence in 2010 and full operational capability is planned for the end of 2013. As with other GNSSs, in particular the widely-used GPS, Galileo signals will be applicable for time and frequency comparisons between distant clocks.

Galileo will provide 4 position, navigation and timing (PNT) services: Open Service, Safety of Life, Commercial Service and Public Regulated Service, as well as a search and rescue support (i.e. communications) service. The PNT services will be carried by 6 groups of signals: E5A and E5B (on either side of $1191.795 \mathrm{MHz})$, E6P and E6C (1278.75 $\mathrm{MHz}), \mathrm{L} 1 \mathrm{P}$ and $\mathrm{L} 1 \mathrm{~F}(1575.42 \mathrm{MHz})$. The E5A and E5B signals may alternatively be considered together as a single, wideband signal, called E5. The ranging signals on E5A, E5B and $\mathrm{L} 1 \mathrm{~F}$ will be unencrypted while the data channel on E5A will be unencrypted and those on E5B and L1F partially encrypted. The E6C, E6P and L1P signals will be encrypted, for commercial and governmental applications respectively.

Thus Galileo is planned to provide 4 freely-accessible single-frequency signals suitable for time and frequency comparisons using the common-view, code-based method: E5A, E5B, E5 and L1F. These signals allow for 6 dual-frequency pairs, of which those between E5 and L1 are certainly the most interesting: E5A-L1F, E5B-L1F, E5-L1F, the others being: E5A-E5B, E5A-E5, E5-E5B, each of which may be used to construct an ionospherefree combination analogous to the well-known "P3" combination of GPS signals. The All in View and Precise Point Positioning (PPP) methods, which play an important role in time and frequency comparisons, will also be applicable to the Galileo signals, subject to the International GNSS Service (IGS) providing Galileo products similar to its current GPS products.

We note that the "CGGTTS" data exchange format, used for common view time and frequency comparisons, already includes identifiers for Galileo satellites but still needs to be extended with identifiers for the different possible Galileo signals and combinations.

We mention also that the Galileo signals allow the possibility of constructing triplefrequency combinations which eliminate both the ionospheric and the geometrical contributions, thus providing an additional tool for studying and mitigating various error terms such as code delays, multi-path, receiver noise and phase ambiguities.

Finally we note that allowing national time metrology laboratories access to the commercial and governmental Galileo signals would certainly improve measurement capabilities, as well as providing valuable feedback to the Galileo system itself. 